\title{
Tuning thermoelectric power factor by crystal-field and spin-orbit couplings in Kondo lattice materials
}

\author{
Seungmin Hong, ${ }^{1}$ Pouyan Ghaemi, ${ }^{1,2,3}$ Joel E. Moore, ${ }^{2,3}$ and Philip W. Phillips ${ }^{1}$ \\ ${ }^{1}$ Department of Physics, University of Illinois at Urbana-Champaign, Urbana, IL 61801, USA \\ ${ }^{2}$ Department of Physics, University of California, Berkeley, CA 94720, USA \\ ${ }^{3}$ Materials Sciences Division, Lawrence Berkeley National Laboratory, Berkeley, CA 94720, USA
}

(Dated:)

\begin{abstract}
We study thermoelectric transport at low temperatures in correlated Kondo insulators, motivated by the recent observation of a high thermoelectric figure of merit(ZT) in $F e S b_{2}$ at $T \sim 10 K^{[1}$. Even at room temperature, correlations have the potential to lead to high $\mathrm{ZT}$, as in $Y b \mathrm{Al}_{3}$, one of the most widely used thermoelectric metals. At low temperature correlation effects are especially worthy of study because fixed band structures are unlikely to give rise to the very small energy gaps $E_{g} \sim 5 k T$ necessary for a weakly correlated material to function efficiently at low temperature. We explore the possibility of improving the thermoelectric properties of correlated Kondo insulators through tuning of crystal field and spin-orbit coupling and present a framework to design more efficient low-temperature thermoelectrics based on our results.
\end{abstract}

\section{INTRODUCTION}

Thermoelectrics support a voltage drop in response to a modest temperature gradient. Since a temperature gradient affects the electrons and the lattice degrees of freedom, optimizing thermoelectrics involves not only the thermopower or Seebeck coefficient $(S)$, but also the electrical $(\sigma)$ and thermal $(\kappa)$ conductivities. The holy grail of thermoelectrics is to achieve a figure of merit

$$
Z T=\left(\frac{S^{2} \sigma}{\kappa}\right) T
$$

that exceeds unity at room temperature. This tall order remains a grand challenge problem ${ }^{2}$ [5. Two promising recent directions have focused on either decreasing the thermal conductivity as in the case of nanocrystalline arrays of $\mathrm{Bi}_{x} \mathrm{Sb}_{2-x} \mathrm{Te}_{3}$ in which a $Z T$ of 1.4 was achieved ${ }^{3}$ at $T=373 K$ or maximizing the numerator of $Z$ through strong electron correlations. An example of the latter is the report ${ }^{1}$ that $\mathrm{FeSb}_{2}$ achieves a colossal thermopower of $45000 \mu \mathrm{V} / \mathrm{K}$ at $10 \mathrm{~K}$ resulting in the largest power factor, $S^{2} \sigma$ witnessed to date. In this paper, we follow-up on the role strong correlations play in maximizing the power factor by focusing on Kondo insulators. We show explicitly that multi-orbital physics in Kondo insulators lies at the heart of the problem of maximizing the power factor.

Because the thermopower is related to the entropy per carrier, particle-hole asymmetry and large density of states at the chemical potential are central to the optimization of $Z$. In this regard, the Anderson model of a single impurity in a metal $\sqrt{6}$, which is among the few solvable strongly correlated systems solvable exactly, presents a density of states with demanding features for efficient thermoelectric transport. For a single $S U(2)$ spin on a localized impurity, the density of states appears as a single infinite symmetric peak at the chemical potential leading to a divergent density of states but vanishing Seebeck coefficient by virtue of the particle-hole symme- try. Increasing the degeneracy of the localized orbital and the metallic band to $S U(N)(N>2)$ softens the peak in the density of states and at the same time moves above the chemical potential leading to an asymmetric density of states and as a result a larger Seebeck coefficient ${ }^{7}$.

It makes sense then to consider systems in which such physics is naturally present, for example Kondo insulators in which a regular lattice of Anderson impurities is hybridized with multiple bands of itinerant electrons. The electrons in the local orbitals are poorly screened and the strong Coloumb repulsion prohibits them from being multiply occupied. Contrary to the single impurity, the periodic Anderson model is not exactly solvable but multiple mean-field type methods were used $\frac{\sqrt[8]{10}}{-10}$ understand many of their features. Motivated by the single impurity model, we examine the effect of degeneracy of the local impurities and the conduction band on the thermoelectric properties of Kondo insulators. In addition to directly studying the degeneracy of the local and conduction bands, we study the effect lifting the degeneracy by a crystal-field (which mainly effects the local orbitals) and spin-orbit coupling (which mainly effects the conduction band) have on thermoelectric efficiency. In this way, we can continuously lift the level of degeneracy. Interestingly, we observe that there is an optimum value of the crystal field and spin orbit coupling. As was shown in a previous study 11 , the presence of multiple orbitals close to the chemical potential is a common feature of Kondo insulators. Our results indeed present a possible route for using strong correlations to enhance the thermoelectric performance through controlling the orbital degeneracy of local and itinerant bands.

\section{MODEL AND METHODOLOGY}

Heavy fermion materials typically contain rare earth or actinide ions forming a lattice of localized magnetic moments 12 . The strong Coulomb repulsion of electrons 
localized in $f$ or $d$ orbitals leads to formation of these local moments ${ }^{\sqrt{6}}$ which then hybridize with the itinerant electron bands and form the heavy electron bands. If the chemical potential is in the heavy electron bands, a heavy fermion metal is formed. The volume of the Fermi surface in this correlated state corresponds to a sum of the number of itinerant and localized electrons. If the chemical potential is in the hybridization gap, the heavy electron band will be fully occupied and a Kondo insulator obtains $53 \mid 14$. Notice that such an insulating state is fundamentally different from a non-interacting insulator. For example, in order to reach a filled valance band, we need to add the number of localized and itinerant electrons which is solely developed as a result of strong interactions.

The underlying microscopic model of this correlated system is:

$$
\begin{aligned}
H= & \sum_{\mathbf{k} l \sigma \sigma^{\prime}} \varepsilon_{\sigma \sigma^{\prime}}(\mathbf{k}) c_{\mathbf{k} l \sigma}^{\dagger} c_{\mathbf{k} l \sigma^{\prime}}+\sum_{\mathbf{k} l \sigma} \epsilon_{f_{l}} d_{\mathbf{k} l \sigma}^{\dagger} d_{\mathbf{k} l \sigma}+ \\
& \sum_{i \mathbf{k} l \sigma}\left(V_{\mathbf{k} l \sigma} e^{i \mathbf{k} \cdot \mathbf{r}_{i}} c_{\mathbf{k} l \sigma}^{\dagger} d_{i l \sigma}+\text { h.c. }\right) \\
& +\frac{U}{2}\left(\sum_{i l, \sigma \neq \sigma^{\prime}} n_{i l \sigma}^{d} n_{i l \sigma^{\prime}}^{d}+\sum_{i, l \neq l^{\prime}, \sigma \sigma^{\prime}} n_{i l \sigma}^{d} n_{i l^{\prime} \sigma^{\prime}}^{d}\right)
\end{aligned}
$$

where $c_{\mathbf{k} l \sigma}^{\dagger}\left(d_{\mathbf{k} l \sigma}^{\dagger}\right)$ is the creation of a conduction(local) electron with momentum $\mathbf{k}$, orbital $l$, and $\operatorname{spin} \sigma=(\uparrow, \downarrow)$, and $n_{i l \sigma}^{d}=d_{i l \sigma}^{\dagger} d_{i l \sigma}$ is the number operator of a local $d$ orbital at site $\mathbf{r}_{i}$. The dispersion of the $c$-electron $\varepsilon_{\sigma \sigma^{\prime}}(\mathbf{k})=\epsilon_{\mathbf{k}} \delta_{\sigma \sigma^{\prime}}+\boldsymbol{\Gamma}_{\mathbf{k}} \cdot \boldsymbol{\sigma}_{\sigma \sigma^{\prime}}$ includes spin coupling. The non-dispersive energy of local states $\left(\epsilon_{f_{l}}\right)$ depends on the orbital index $l$. The pseudovector $\boldsymbol{\Gamma}_{\mathbf{k}}$ represents the amplitude of the spin-orbit (SO) coupling $15 \mid 16$ and its form depends on the crystal symmetry of the underlying lattice (see appendix A). Typically, the hybridization matrix element, $V_{\mathbf{k} l \sigma}$ encodes the complex orbital structures of local states which can have novel effects on the properties of the strongly correlated heavy fermion phase 17 , but as in other studies, we consider $V_{\mathbf{k} l \sigma}$ to be independent of $(\mathbf{k}, \sigma)$ to make the calculation more tractable.

Using the model Hamiltonian, Eq. (2), we can capture the effect of the degeneracy of both localized and itinerant bands, as well as the effect of crystal field and spin orbit coupling in breaking the degeneracy of these bands. As a result of weak screening of electrons in $f$ and $d$ orbitals, the associated on-site repulsive potential $U$ is much larger than the hopping energies of the itinerant electrons. To treat the large on-site repulsion term, we use the $U(1)$ slave-boson mean-field theory 9118 . In this treatment, the creation operator of a local electron $d_{i l \sigma}^{\dagger}=f_{i l \sigma}^{\dagger} b_{i}$ is partitioned into a neutral fermion $f_{i l \sigma}^{\dagger}$, and a charged boson $b_{i}$ that accounts for annihilation of an empty state. Since the local Hilbert space is restricted to either an empty or a singly occupied state, the additional local constraint,

$$
\tilde{Q}_{i}=b_{i}^{\dagger} b_{i}+\sum_{l \sigma} f_{i l \sigma}^{\dagger} f_{i l \sigma}=1
$$

should be enforced at every site $\mathbf{r}_{i}$. The Hamiltonian in terms of these slave particles then becomes

$$
\begin{aligned}
H= & \sum_{\mathbf{k} l \sigma \sigma^{\prime}} \varepsilon_{\sigma \sigma^{\prime}}(\mathbf{k}) c_{\mathbf{k} l \sigma}^{\dagger} c_{\mathbf{k} l \sigma^{\prime}}+\sum_{\mathbf{k} l \sigma} \epsilon_{f_{l}} f_{\mathbf{k} l \sigma}^{\dagger} f_{\mathbf{k} l \sigma} \\
& +\sum_{i \mathbf{k} l \sigma}\left(V_{l}^{*} e^{-i \mathbf{k} \cdot \mathbf{r}_{i}} f_{i l \sigma}^{\dagger} b_{i} c_{\mathbf{k} l \sigma}+\text { h.c. }\right)+\sum_{i} \lambda_{i}\left(\tilde{Q}_{i}-1\right)
\end{aligned}
$$

where $\lambda_{i}$ is a Lagrange multiplier to maintain the local constraint. In the above Hamiltonian, the effect of the crystal field is to break the degeneracy of the local orbital states $\epsilon_{f_{l}}$ whereas the spin-orbit coupling breaks the spin degeneracy of the conduction band. As a result, by tuning the crystal field and spin-orbit coupling, we can change the degeneracy of the local and conduction orbitals in a continuous manner. Consequently, we have a tunable knob to gain the optimum thermoelectric performance.

The mean field approximation to the model Hamiltonian can be obtained by taking the coherent expectation $b=\left\langle b_{i}\right\rangle=\left\langle b_{i}^{\dagger}\right\rangle$ and $\lambda=\left\langle\lambda_{i}\right\rangle$. This replacement effectively renormalizes the mixing matrix element $V_{l} \rightarrow b V_{l}$, and the local energy $\epsilon_{f_{l}} \rightarrow \epsilon_{f_{l}}+\lambda$ and leads to the quadratic Hamiltonian

$$
\begin{aligned}
H_{\mathrm{MF}} & =\sum_{\mathbf{k} l h}\left(\epsilon_{\mathbf{k}}+h\left|\boldsymbol{\Gamma}_{\mathbf{k}}\right|\right) c_{\mathbf{k} l h}^{\dagger} c_{\mathbf{k} l h}+\sum_{\mathbf{k} l \sigma}\left(\epsilon_{f_{l}}+\lambda\right) f_{\mathbf{k} l h}^{\dagger} f_{\mathbf{k} l h} \\
& +\sum_{\mathbf{k} l h}\left(b V_{l}^{*} f_{\mathbf{k} l h}^{\dagger} c_{\mathbf{k} l h}+\text { h.c. }\right)+\lambda \sum_{i}\left(b^{2}-1\right)
\end{aligned}
$$

Instead of working in the spin basis, we will use a helical basis that diagonalizes the single-electron dispersion $\varepsilon_{\sigma \sigma^{\prime}}(\mathbf{k}) \rightarrow\left[U_{\mathbf{k}}^{\dagger} \varepsilon(\mathbf{k}) U_{\mathbf{k}}\right]_{h h^{\prime}}=\left(\epsilon_{\mathbf{k}}+h\left|\boldsymbol{\Gamma}_{\mathbf{k}}\right|\right) \delta_{h h^{\prime}}$ with $h, h^{\prime}= \pm 1$. Then $c_{\mathbf{k} l h}\left(f_{\mathbf{k} l h}\right)$ is accordingly rotated by the unitary matrix $U_{\mathbf{k}}$ from the spin basis, $c_{\mathbf{k} l \sigma}\left(f_{\mathbf{k} l \sigma}\right)$. By performing the Bogoliubov transformation,

$$
\begin{aligned}
& a_{\mathbf{k} l h+}=\alpha_{\mathbf{k} l h} c_{\mathbf{k} l h}+\beta_{\mathbf{k} l h} f_{\mathbf{k} l h} \\
& a_{\mathbf{k} l h-}=-\beta_{\mathbf{k} l h} c_{\mathbf{k} l h}+\alpha_{\mathbf{k} l h} f_{\mathbf{k} l h},
\end{aligned}
$$

we obtain the diagonal mean-field Hamiltonian,

$$
H_{\mathrm{MF}}=\sum_{\mathbf{k} l h, \pm} E_{\mathbf{k} l h}^{ \pm} a_{\mathbf{k} l h \pm}^{\dagger} a_{\mathbf{k} l h \pm}+\lambda \sum_{i}\left(b^{2}-1\right),
$$

where the dispersion is given by

$$
\begin{aligned}
E_{\mathbf{k} l h}^{ \pm} & =\frac{1}{2}\left(\epsilon_{\mathbf{k}}+h\left|\boldsymbol{\Gamma}_{\mathbf{k}}\right|+\epsilon_{f_{l}}+\lambda \pm W_{\mathbf{k} l h}\right), \\
W_{\mathbf{k} l h} & =\sqrt{\left(\epsilon_{\mathbf{k}}+h\left|\boldsymbol{\Gamma}_{\mathbf{k}}\right|-\epsilon_{f_{l}}-\lambda\right)^{2}+4 b^{2} V_{l}^{2}} .
\end{aligned}
$$

The Bogoliubov parameters are

$$
\left(\begin{array}{c}
\alpha_{\mathbf{k} l h}^{2} \\
\beta_{\mathbf{k} l h}^{2}
\end{array}\right)=\frac{1}{2}\left[1 \pm \frac{\left(\epsilon_{\mathbf{k}}+h\left|\boldsymbol{\Gamma}_{\mathbf{k}}\right|\right)-\left(\epsilon_{f_{l}}+\lambda\right)}{W_{\mathbf{k} l h}}\right] .
$$


Minimization of the free energy with respect to the mean field parameters $b$ and $\lambda$, and the total chemical potential $\mu$ leads to two coupled equations

$$
\begin{aligned}
1 & =b^{2}+\sum_{\mathbf{k} l h} \alpha_{\mathbf{k} l h}^{2} n_{F}\left(E_{\mathbf{k} l h+}\right)+\beta_{\mathbf{k} l h}^{2} n_{F}\left(E_{\mathbf{k} l h-}\right) \\
\lambda & =\sum_{\mathbf{k} l h} \frac{V_{l}^{2}}{W_{\mathbf{k} l h}}\left[n_{F}\left(E_{\mathbf{k} l h-}\right)-n_{F}\left(E_{\mathbf{k} l h+}\right)\right] \\
n_{\text {tot }} & =\sum_{\mathbf{k} l h}\left[n_{F}\left(E_{\mathbf{k} l h-}\right)+n_{F}\left(E_{\mathbf{k} l h+}\right)\right]
\end{aligned}
$$

where, the total density of electrons is fixed to be $n_{\text {tot }}=$ $2 l_{\max }$ for $l=1,2, \cdots, l_{\max }$. The transport of this noninteracting mean-field Hamiltonian is now tractable.

To compute the transport properties, we use the relaxation-time approximation to the Boltzmann equation 19. Under this scheme, the electrical resistivity, $\boldsymbol{\rho}=\boldsymbol{\sigma}^{-1}$, and the thermopower tensors, $\mathbf{S}$, are given by

$$
\boldsymbol{\rho}=\mathbf{L}_{0}^{-1}, \quad \mathbf{S}=-\frac{k_{B}}{|e|} \mathbf{L}_{0}^{-1} \mathbf{L}_{1},
$$

where the tensors $\mathbf{L}_{m}$ are

$$
\begin{aligned}
\left(\mathbf{L}_{m}\right)_{a b}= & -\frac{e^{2}}{V_{\text {olume }}} \sum_{\mathbf{k} l h \pm} \frac{\partial n_{F}\left(E_{\mathbf{k} l h \pm}\right)}{\partial E_{\mathbf{k} l h \pm}} \\
& \times \tau_{\mathbf{k} l h \pm}\left(\mathbf{v}_{\mathbf{k} l h \pm}\right)_{a}\left(\mathbf{v}_{\mathbf{k} l h \pm}\right)_{b}\left(\frac{E_{\mathbf{k} l h \pm}-\mu}{k_{B} T}\right)^{m}
\end{aligned}
$$

explicitly. Here we set $\mathbf{v}_{\mathbf{k} l h \pm}=\frac{1}{\hbar} \nabla E_{\mathbf{k} l h \pm}$. Considering that the electrons are scattered by $N_{\text {imp }}$ impurities with an interaction strength of $V_{\text {imp }}$ (i.e., $H_{\text {sctt }} \sim$ $\left.V_{\mathrm{imp}} c_{\mathbf{k}^{\prime} l \sigma}^{\dagger} c_{\mathbf{k} l \sigma}\right)$, the relaxation time $\tau_{\mathbf{k} l h \pm}$ for each state is given by

$$
\frac{1}{\tau_{\mathbf{k} l h \pm}}=\frac{2 \pi}{\hbar} \frac{N_{\mathrm{imp}}}{N_{\text {site }}}\left|V_{\mathrm{imp}}\right|^{2}\left[\frac{\partial E_{\mathbf{k} l h \pm}}{\partial\left(\epsilon_{\mathbf{k}}+h\left|\boldsymbol{\Gamma}_{\mathbf{k}}\right|\right)}\right]^{2} \rho_{l h}\left(E_{\mathbf{k} l h \pm}\right),
$$

with $\rho_{l h}\left(E_{\mathbf{k} l h \pm}\right)$ the density of the states of the Bogoliubov quasiparticles.

\section{RESULTS}

We now present our results on the dependence of the transport properties on the orbital degeneracies of both localized and itinerant electron bands which form correlated Kondo insulators. In the first two subsections, we consider double degeneracy of conduction and localized bands. This model is indeed consistent with the models previously proposed for Kondo insulators 20 . The crystal field will then split the degeneracy of the two $f$ levels in 4 and spin-orbit coupling breaks the degeneracy of the conduction band states with differing helicity 16 . In these two sections, we change the size of the degeneracybreaking gap continuously. Using the relaxation-time approximation, we can then calculate the transport properties of a Kondo insulator. For most of the materials, the dominant contribution to the thermal conductivity comes from lattice vibrations; as a consequence, the electronic contribution to the thermoelectric performance is measured through the power factor $Z_{\mathrm{PF}}=\sigma S^{2}$ where $\sigma$ is the electrical conductivity and $S$ is the Seebeck coefficient. In order to confirm that the enhancement of the thermoelectric efficiency can properly be attributed to strong correlations, we consider two different band structures of itinerant electrons in $\amalg \mathrm{A}$ and $\amalg \mathrm{IIB}$ and we see that similar features emerge.

Finally, in III C we present the effect of multiple orbital degeneracy. Contrary to the treatment in III A and III B where the double degeneracy is continuously lifted by crystal field and spin-orbit couplings, in section [IIC we discretely change the number of degenerate conduction and localized bands. We show that indeed there is also an optimum degeneracy associated with the maximum power factor.

\section{A. Nearly free electron itinerant bands}

We first focus on the effect of crystal field and spinorbit coupling on the power factor within the context of a parabolic band for the itinerant electrons $\epsilon_{\mathbf{k}}=$ $\epsilon_{0}+W\left(k / k_{B Z}\right)^{2}$ with $W=2 \mathrm{eV}$, taken from ref. ${ }^{21}$. In principle, the SO coupling should be expressed as a periodic function under the crystal environment, but we model it to be isotropic as well, $\boldsymbol{\Gamma}_{\mathbf{k}}=\gamma_{\mathrm{so}}\left(\mathbf{k} / k_{B Z}\right)$ $\left(\gamma_{\mathrm{so}} \leq 0.2 \mathrm{eV}\right)$. In the following, we carry out the numerical calculation based on this isotropic band dispersion with $l_{\max }=2$. Here, we choose $\epsilon_{f_{1}}=1.0606 \mathrm{eV}$, $V_{1}=0.2236 \mathrm{eV}$ and $V_{2}=1.05 V_{1}=0.2348 \mathrm{eV}$. The parameter $\frac{N_{\text {imp }}}{N_{\text {site }}}\left|V_{\text {imp }}\right|^{2}=0.045 \mathrm{eV}^{221}$. The other control parameters are temperature $(T \leq 100 K)$, crystal electric field (CEF) splitting $\left(\Delta_{\mathrm{CEF}}=\epsilon_{f_{2}}-\epsilon_{f_{1}} \leq 15 \mathrm{meV}\right)$, and the SO coupling $\left(\gamma_{\mathrm{so}} \leq 0.15 \mathrm{eV}\right)$. Although we do not include the supporting data here, we found that our conclusions are insensitive to the strength of $V_{2}$ as long as $0.5 \lesssim V_{2} / V_{1} \lesssim 2.0$.

Figure 1 shows the density of states (DOS) for different control parameters. From Figs. 1(a) to (d), we notice that the temperature only controls the number of thermally activated charge carriers, while it does not significantly change the DOS compared to the other parameters. When the degeneracy of the two local orbitals is broken by the crystal electric field, one of the hybridized bands moves closer to the chemical potential. Consequently, the system is driven from an insulator to a conductor [Figs. 11(e)-(h)], at which point the power factor is significantly enhanced (See Fig. 2). Likewise, the spinorbit interaction breaks the degeneracy of the two helical modes, which turns an insulator into a metallic state [Figs. 1(i)-(l)]. We point out that the metallic state is characterized either by a local orbital, $l=2$ [Fig. 1(h)] or by a helicity, $h=+[$ Fig. 1 $1(1)]$, since only the bands with corresponding quantum numbers are conducting.

In order to further examine the effect of the control 


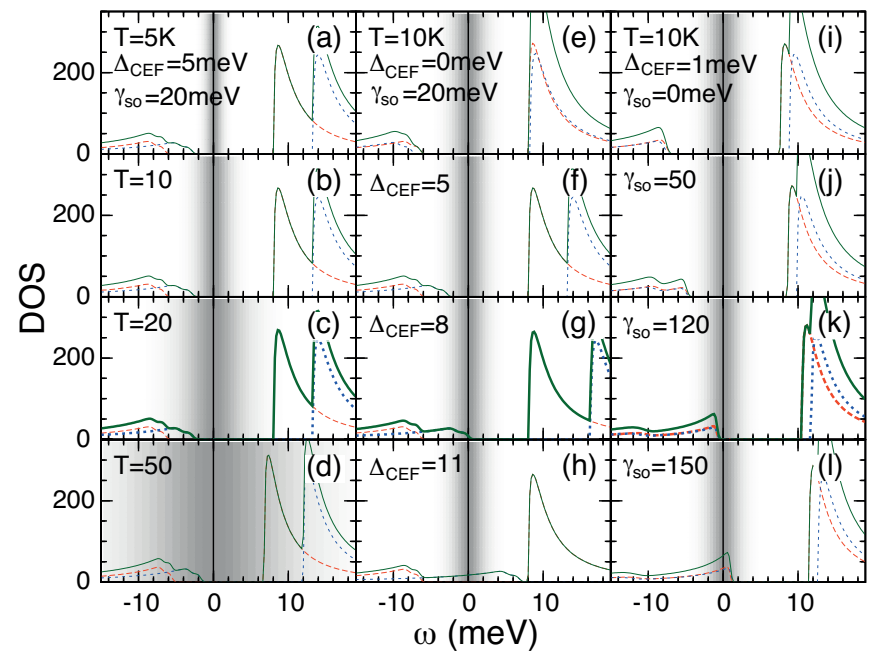

FIG. 1. Density of states (DOS) of the parabolic model for different control parameters. The red-dashed lines are for the orbital $l=1$, the dotted blue lines $l=2$, and the green lines are for the total DOS. The central gray area displays the thermal window $\left(\sim k_{B} T\right)$ for each temperature. Figures (a)-(d) compare the DOS for different temperatures. Figures (e)-(h) correspond to different magnitudes of the crystal electric field $\left(\Delta_{\mathrm{CEF}}\right)$ which breaks the degeneracy of the two $f$ orbitals. Figures (i)-(l) correspond to different magnitudes of the spin-orbit interaction $\left(\gamma_{\mathrm{SO}}\right)$ that breaks the degeneracy of the conduction bands with different helicity.

parameters, we first calculate the transport coefficients as a function of the CEF and SO. In Fig. 2, we show the results of a calculation of the thermopower $(S)$, the electrical conductivity $(\sigma)$, and the power factor $\left(Z_{\mathrm{PF}}\right)$. As can be seen from the right column, the power factor is enhanced either by finding the optimal CEF or by adjusting the SOI. Since both CEF and SOI shift some of the lower energy bands toward the chemical potential [Figs. 1(e)-(l)], the number of lower energy bands relevant for thermal transport is controlled by CEF and SOI simultaneously. For the temperature range $T \lesssim 20 K$, where the thermal windows are sufficiently narrow, CEF and SOI compete; hence there are two distinctive optimal regimes. For a sufficiently wide thermal window, attainable at intermediate temperatures, $T \sim 30 K$, CEF and SOI are working cooperatively to form a single optimal region. For $T>30 K$, the enhancement in $Z_{\mathrm{PF}}$ is not as drastic as at low temperature. $Z_{\mathrm{PF}}$ is maximized in the vicinity of the insulator-metal transition (see the conductivity $\sigma$ at $T=5-20 \mathrm{~K}$ ), resulting from a competition between $S$ and $\sigma$. For instance, at $T=5 K$, the thermopower $S$ decreases with SOI and CEF, while the system acquires a finite conductivity. Note that the metallic state here has one dominant helical state over the other.

In Fig. 3, we repeat the calculation of the transport coefficients for a fixed SOI as a function of CEF and temperature. Consistent with Fig. 2 is that the optimal
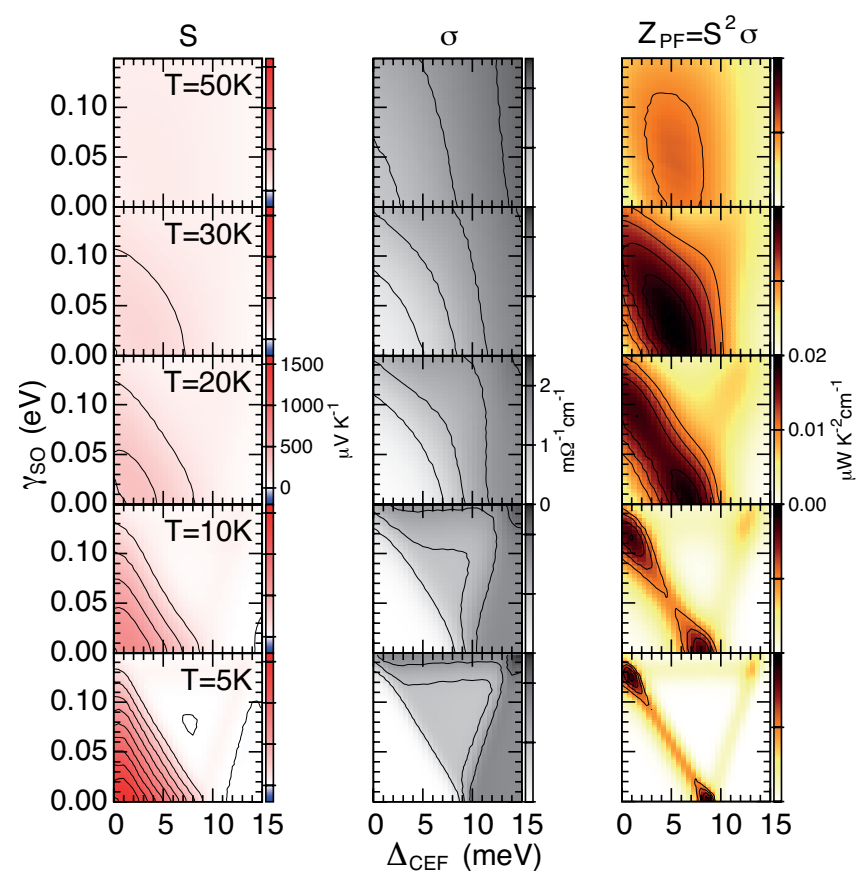

FIG. 2. Transport coefficients for different temperatures as a function of crystal field splitting and spin-orbit interaction: thermopower $S$ (left column), conductivity $\sigma$ (middle), and power factor $Z_{\mathrm{PF}}$ (right). From the top to the bottom, the temperature varies from 50 to $5 \mathrm{~K}$, and the solid lines are equally spaced constant contours.

point for the power factor is located in the vicinity where the insulator-metal transition occurs. For instance, when $\gamma_{\mathrm{so}} \lesssim 0.1 \mathrm{eV}$, there is the optimal CEF and temperature for the power factor, at which point the electric conductivity acquires a noticeable finite value. From the left column, one finds that the thermopower generally decreases with increasing temperature as a widened thermal widow implies the reduction of the asymmetry in the DOS within the thermal region [Figs. 11(a)-(d)]. This obtains because as the temperature increases, more of the bands (lower and upper) are involved in the thermal transport. In other words, the asymmetry of the DOS within the thermally active region is relieved. Beyond a certain of the threshold SOI, $\gamma_{\mathrm{so}} \geq 0.13 \mathrm{eV}$, there is no phase transition (at mean field level); hence optimization cannot be realized.

\section{B. Tight binding itinerant electron bands}

Next, we consider the 3 -dimensional tight binding case. We see that as in the case of a quadratic band, tuning the crystal-field and spin-orbit coupling can optimize the thermoelectric performance. This result indicates that the effect of orbital degeneracy in controlling the thermoelectric performance is not that sensitive to the details of the band structure. Here, we choose the hopping 


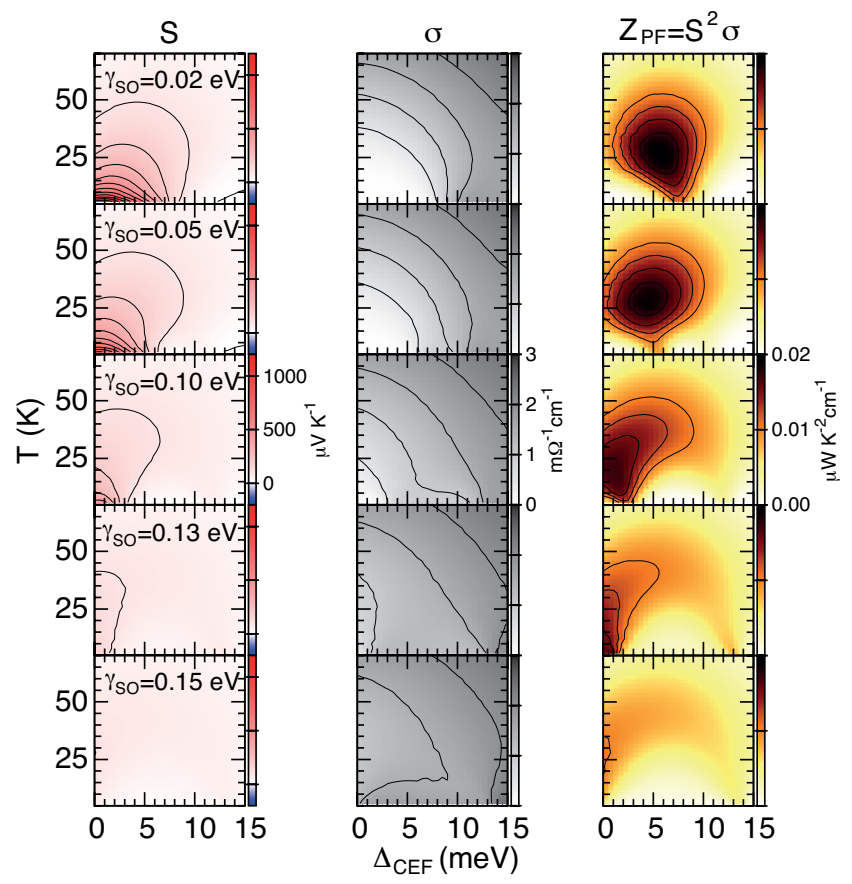

FIG. 3. Transport coefficients for each SOI as a function of $\mathrm{CEF}$ and temperature: thermopower $S$ (left column), conductivity $\sigma$ (middle), and power factor $Z_{\mathrm{PF}}$ (right). The range of $\mathrm{SOI}$ is $0.02-0.15 \mathrm{eV}$ from the top to bottom panels.

parameter $t_{\text {hop }}=0.2167 \mathrm{eV}$ (band width $W=2.6 \mathrm{eV}$ ), and we located the local energy $\epsilon_{f_{1}}=-0.8 t_{\text {hop }}$. The hybridization strength $V_{1}=t_{\text {hop }}$ and $V_{2}=1.01 t_{\text {hop }}$. In the SOI, we take the next-nearest neighbor hopping parameter $g_{2}=0.3$.

As in the simplified parabolic model, the role of CEF and SOI is not different; both efficiently control the system to drive it from an insulator to a conductor as seen from Fig. 4. Compared to the corresponding panels in Figs. 11 however, Figs. 4(e)-(h) show that the CEF also pushes one of the upper bands toward the chemical potential, hence reducing the gap size significantly. In fact, the parabolic model is rather exceptional since the bottom of the upper bands corresponds to the point $\mathbf{k}=\mathbf{0}$, which is not usual for typical 3D tight-binding models. Figs. 4(i)-(l) display the evolution of DOS with the increase of the SOI. Even though the degeneracy of the two helical modes are broken with a finite SOI, it cannot be seen clearly as was in the linearized SOI case [Figs. 11(i)(l))]. The reason is that $\left|\boldsymbol{\Gamma}_{\mathbf{k}}\right|$ decreases as $\mathbf{k}$ approaches the boundary of the Brillouin zone due to the periodic form of the SOI, while it does not for the linearized SOI. Unlike the CEF which affected drastically only one of the orbitals, the effect of the SOI is quite different. Up to $\gamma_{\mathrm{so}}=0.2 t_{\mathrm{hop}} \simeq 40 \mathrm{meV}$, the changes in the DOS are not significant. For $\gamma_{\mathrm{so}} \gtrsim 0.2 t_{\text {hop }}$, the system undergoes a phase transition to a (helically polarized) metal, beyond which point $Z_{\mathrm{PF}}$ is reduced (See Fig. 5).

From Fig. 5 we observe consistency with the parabolic model: the power factor can be enhanced by adjusting the CEF, while the SOI slightly lowers the optimal value of the CEF. For $T>15 K$, the enhancement in $Z_{\mathrm{PF}}$ is not as drastic as was in the low temperature case. As in the parabolic model, this trend occurs because the thermally active region is too wide to encompass only one band [see Fig. 4(c) and (d)]. Comparison with the other columns reveals that $Z_{\mathrm{PF}}$ is also maximized near an insulator to a (helical) metal transition (see the conductivity $\sigma$ at $T=5 K$ ), which is the consequence of the competition between $S$ and $\sigma$. Here, one can observe that $S$ becomes maximal at $\Delta_{\mathrm{CEF}} \simeq 0.01 t_{\mathrm{hop}}$, which is a consequence of the choice $V_{2} / V_{1}=1.01$. With $V_{2} / V_{1}=1, S$ only decreases with CEF (not shown). Fig. 6 similarly confirms the consistency with the parabolic model. The only difference is that the effect of SOI is not as remarkable, though it works to shift the optimal value of the CEF. The reason mainly lies in the changes of the DOS depending on SOI: linearized SOI changes the bandwidth significantly, while 3D tight-binding SOI does not due to its periodic structure. (See Fig. 7)

\section{Effect of multi-orbital degeneracy}

In addition to the continuous control of orbital degeneracy through crystal-field and spin-orbit coupling, we can specifically study the effect of increasing the number of degenerate orbitals $\left(l_{\max }=1,2, \cdots, 5\right)$. To minimize the number of free parameters, we will set the orbital de-

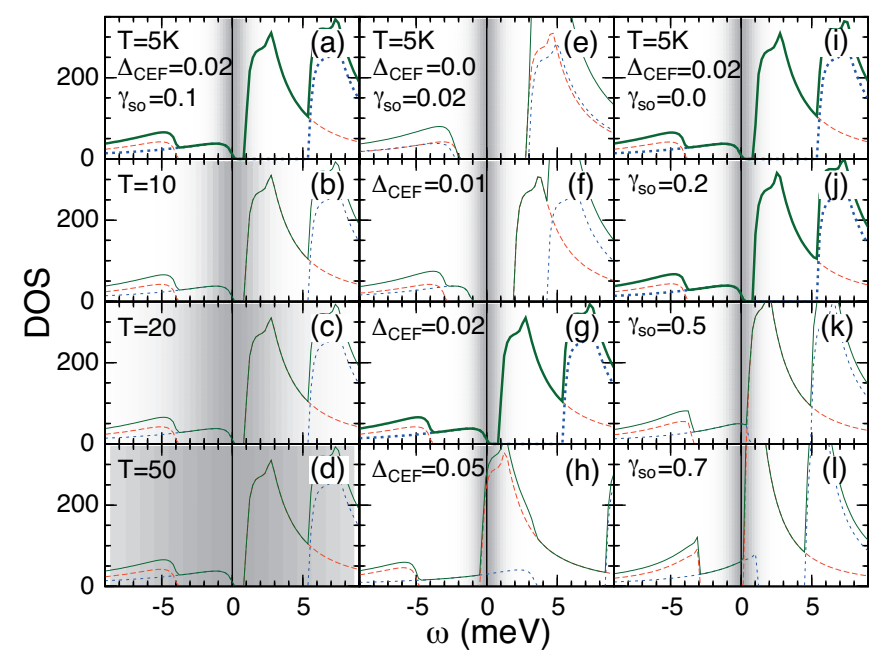

FIG. 4. Density of states(DOS) of the 3D tight-binding model for different control parameters. Both $\Delta_{\mathrm{CEF}}$ and $\gamma_{\mathrm{so}}$ are in units of the hoping amplitude, $t_{\text {hop }}=0.216 \mathrm{eV}$. The reddashed lines are for the orbital $l=1$, the dotted blue lines are for $l=2$, and the green lines are for the total DOS. The central gray area indicates the thermally active region for each temperature. Figs. (a)-(d) compare the DOS for different temperature, (e)-(h) for the crystal electric field $\left(\Delta_{\mathrm{CEF}}\right)$, and (i)-(l) for the spin-orbit interaction $\left(\gamma_{\mathrm{SO}}\right)$. 


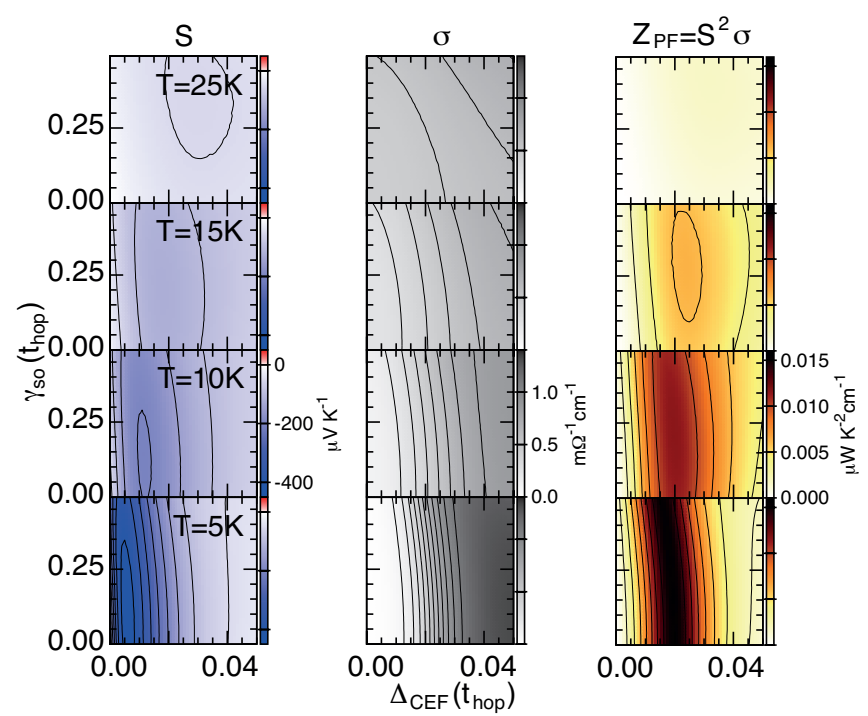

FIG. 5. Transport coefficients for each temperature: thermopower $S$ (left column), conductivity $\sigma$ (middle), and power factor $Z_{\mathrm{PF}}$ (right). From the top to the bottom, the temperature is fixed to $25,15,10$, and $5 \mathrm{~K}$, respectively.

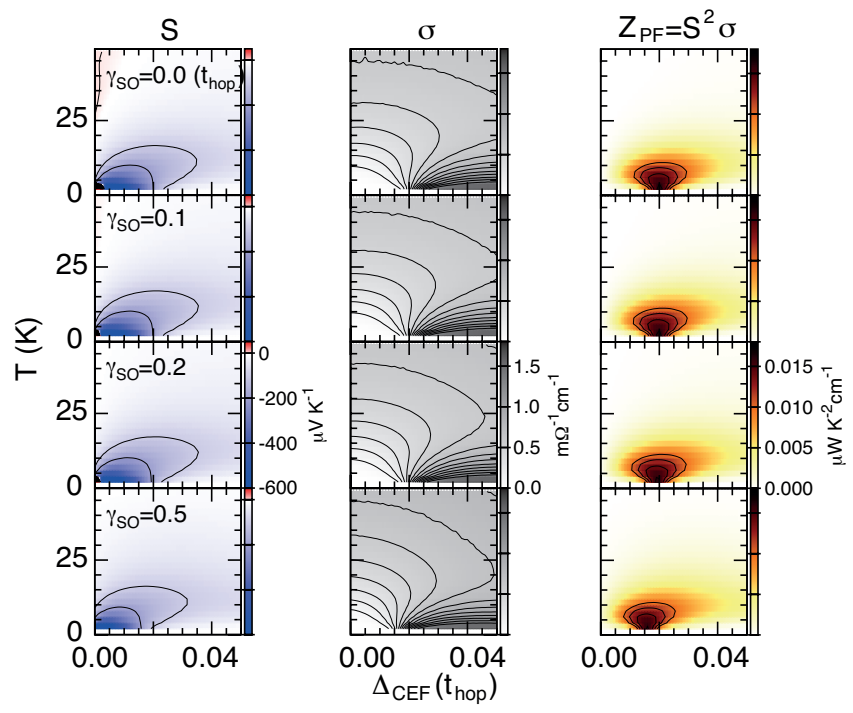

FIG. 6. Transport properties are compared for each SOI: $S$ (left column), conductivity $\sigma$ (middle), and power factor $Z_{\mathrm{PF}}$ (right). The spin-orbit couplings are chosen to be 0.0, 0.1, 0.2, and 0.5 in units of the hoping amplitude $t_{\text {hop }}$, respectively.

generacy of the two bands (the allowed values of $l$ ) to be equal. Although continuous control is not possible in this case, one can then consider changing the material content to achieve a better thermoelectric. Here, the bare conduction electron dispersion is taken to be that of the 3D tight binding model.

First, we compare the DOS depending on the number of orbitals involved [Fig. 8]. As $l_{\max }$ increases, the asym-
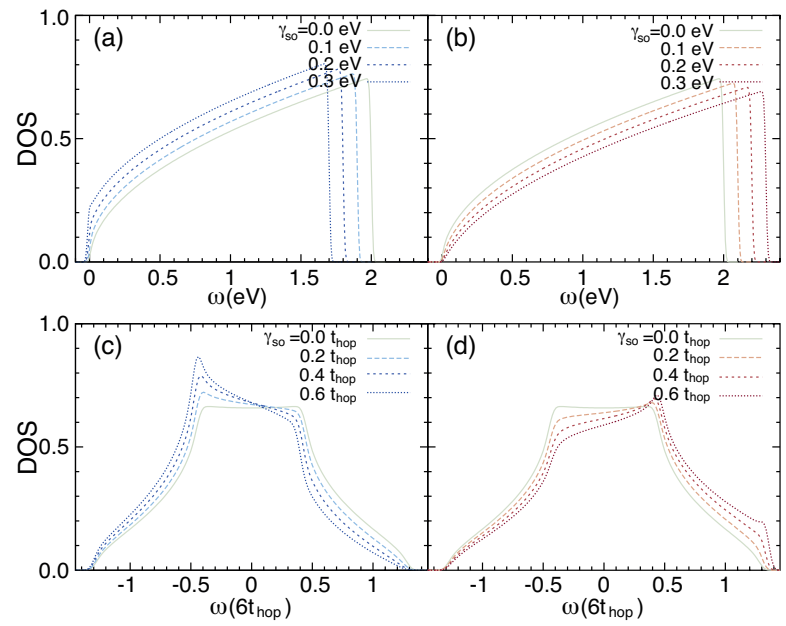

FIG. 7. Density of states for bare conduction electrons: quadratic dispersion(top), 3-D tight binding(bottom), helicity $h=-1$ (left, blue curves) and $h=1$ (right, red lines). For the quadratic dispersion, the SOI is taken to be linear in momentum, as $\gamma_{\mathrm{so}}|\mathbf{k}|$. The changes in the bandwidth are exactly proportional to $\gamma_{\mathrm{so}}$. In figures (c) and (d), the SO term, $\gamma_{\mathrm{so}} \boldsymbol{\Gamma}_{\mathbf{k}}$ is taken in accordance with the cubic point group symmetry, and the next nearest neighbor hopping parameter $g_{2}=0.3$. Note that the bandwidths are not drastically affected by the $\mathrm{SO}$, while the shapes become more asymmetric with the increase in $\gamma_{\text {so }}$.

metry between the upper and the lower bands becomes more pronounced. At the same time, the insulating gap increases with $l_{\max }$ for $l_{\max } \geq 2$. Note that this feature is quite similar to the single impurity problem with $N$ flavors. The inset of Fig. 8 displays the DOS without adjusting the chemical potential. Since the slave-boson method renormalizes the local energy by $\epsilon_{f} \rightarrow \epsilon_{f}+\lambda$, the relative location of the Kondo resonance for each case (near each gap) indicates that the amount of renormalization $\lambda$ increases with the number of available orbitals. Since each band below and above the insulating gap should accommodate one electron, the deformation of the lower bands becomes less significant as $l_{\max }$ increases (see the lower bands for different $l_{\max }$ ). In other words, since the area below and above the gap should be equal, the asymmetry of the DOS becomes more significant as $\lambda$, or equivalently $l_{\max }$, increases.

Given the band structures at the mean-field level, we proceed to calculate the transport properties as shown in Fig. 9. As $l_{\max }$ increases, maxima of $Z_{\mathrm{PF}}$ also increases as the temperature is elevated. The thermopower is also enhanced with the number of available orbitals, which is caused by pronounced asymmetry in the DOS (See Fig. 8). Since the gap size increases, the conductivity generally decreases with the number of orbitals for $l_{\max } \geq 2$. Though not shown here, the maximal power factor per orbital, $Z_{\mathrm{PF}} / l_{\max }$, also increases with $l_{\max }$ until $l_{\max }<7$. In Fig. 9(c), the thermal conductivity due to electronic structure is evaluated, exclud- 


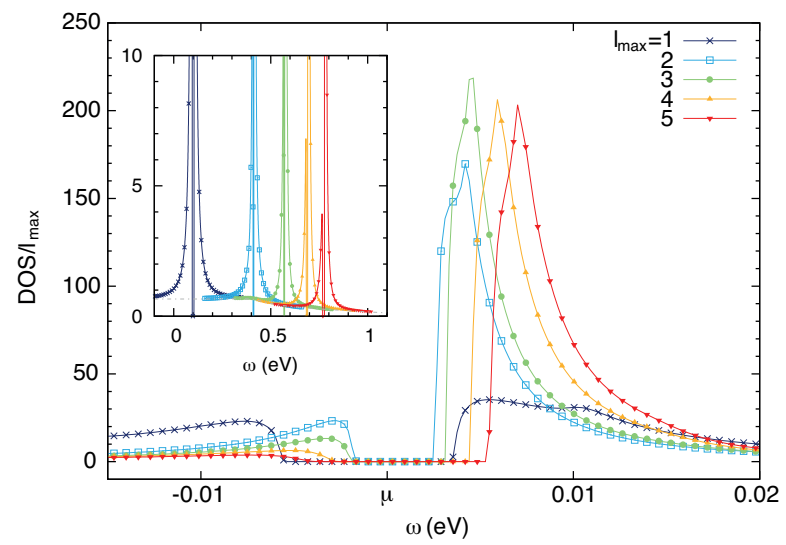

FIG. 8. Density of states per orbital for different number of orbitals $\left(l_{\max }\right)$, where the chemical potential at each case is adjusted to the center. The inset displays the DOS without the adjustment.
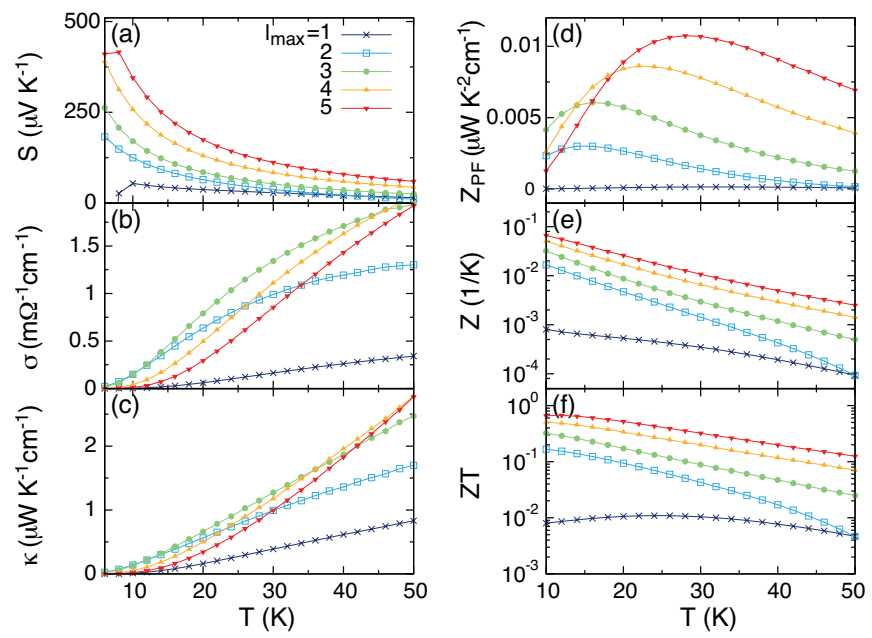

FIG. 9. Transport properties for $l_{\max }$ orbitals: (a) Seebeck coefficient, (b) electrical conductivity, (c) thermal conductivity, (d) thermopower (e) figure of merit and (f) dimensionless figure of merit.

ing any contribution from lattice vibrations. Typically, phonons are dominant contributors to the thermal conductivities, but it may not be so prevalent at the low temperature range considered here, presumably $T \lesssim 10 \mathrm{~K}$. The resultant (dimensionless) figure of merit, assuming $\kappa=\kappa_{\text {electron }}+\kappa_{\text {phonon }} \simeq \kappa=\kappa_{\text {electron }}$, is strongly enhanced with the number of orbitals at least by an order of 10 . This is one of the key results of this paper.

\section{SUMMARY}

We have shown that orbital degeneracy opens a new window into increasing the power factor for strongly interacting thermoelectrics. Our key findings are that the power factor is maximized in strongly correlated systems by tuning 1) the gap between nearly degenerate local f-orbitals through the crystal field effect, 2) ) the gap between nearly degenerate itinerant electron bands through the spin-orbit coupling, and 3) the the number of degenerate local and itinerant orbitals.

This approach provides a new parameter space for the design of strongly correlated thermoelectric materials. Our result was derived using the slave-particle mean-field theory, which is not expected to be quantitatively reliable but should capture general trends. The effect of degeneracy in enhancing thermoelectric performance of strongly correlated systems could also be investigated using other methods such dynamical mean-field theory ${ }^{22}$ and finite frequency methods 23 . Another direction for future work is to consider more profound effects of spin-orbit coupling combined with correlations, as in the proposed "topological Kondo insulators" 24, whose surface states are currently being sought experimentally; such surface states have the potential to increase thermoelectric performance at low temperatures ${ }^{2}$.

Finally, a direction that is difficult theoretically but may be important for actual materials is to find ways of interpolating between the effectively itinerant calculation here (i.e., there are plane-wave states of the slave particles) with the "atomic limit",25, where the effects of multiple orbitals have also been considered ${ }^{26}$. The atomic limit, which is valid when the hopping is the smallest energy scale in the problem, has been argued to be relevant to experiments on sodium cobaltates near room temperature 2728. The results of this paper should motivate continued investigation of correlated materials for thermoelectricity and suggest that a guided search with controlled crystal-field splitting may lead to further improvements in thermoelectric figure of merit, especially in the low-temperature regime.

\section{ACKNOWLEDGMENTS}

This work was supported by the U.S. Department of Energy, Office of Basic Energy Sciences, Materials Sciences and Engineering Division, under Contract No. DEAC02-05CH11231 (P.G. and J.E.M.). P.G. also acknowledge support from NSF DMR-1064319. S.H. and P.W.P. are funded by NSF-DMR-1104909.

\section{Appendix A: Spin-orbit coupling}

In the presence of SO coupling $\frac{\sqrt{15} \mid 16}{16}$, the conduction electron dispersion matrix $\varepsilon_{\sigma \sigma^{\prime}}(\mathbf{k})$ is given by

$$
\varepsilon_{\sigma \sigma^{\prime}}(\mathbf{k})=\epsilon_{\mathbf{k}} \delta_{\sigma \sigma^{\prime}}+\boldsymbol{\Gamma}_{\mathbf{k}} \cdot \boldsymbol{\sigma}_{\sigma \sigma^{\prime}}
$$

where $\epsilon_{\mathbf{k}}$ is the dispersion without the SO interaction, and $\boldsymbol{\sigma}$ are the Pauli matrices. (The SO interactions considered here originate from the absence of an inversion symmetry in the crystal lattice.) The antisymmetric SO 
coupling is described by the real pseudovector $\boldsymbol{\Gamma}_{\mathbf{k}}$, which is determined by the point group symmetry of the crystal. For instance, $\mathrm{CePt}_{3} \mathrm{Si}, \mathrm{CeRhSi}_{3}$ and $\mathrm{CeIrSi}_{3}$ belong to tetragonal point group $\left(\mathbb{G}=C_{4 v}\right)$ in which

$$
\begin{aligned}
\boldsymbol{\Gamma}_{\mathbf{k}}=\gamma_{\mathrm{so}} & \left(\hat{\mathbf{k}}_{x} \sin k_{y} a-\hat{\mathbf{k}}_{y} \sin k_{x} a\right. \\
& +\hat{\mathbf{k}}_{z} g_{2} \sin k_{x} a \sin k_{y} a \sin k_{z} c\left(\cos k_{x} a-\cos k_{y} a\right),
\end{aligned}
$$

in the next nearest neighbor approximation for a real $\gamma_{\text {so }}$, the lattice spacing $a, c$ and the next nearest neighbor parameter $g_{2}$. In case of the cubic point group symmetry
$(\mathbb{G}=O)$, the pseudovector is given by

$$
\begin{array}{r}
\boldsymbol{\Gamma}_{\mathbf{k}}=\gamma_{\mathrm{so}} \hat{\mathbf{k}}_{x} \sin k_{x} a\left[1-g_{2}\left(\cos k_{y} a+\cos k_{z} a\right)\right] \\
+(\text { Positive permutations of } x, y, z)
\end{array}
$$

In real noncentrosymmetric crystals, the typical SO strength ranges up to $200 \mathrm{meV}$. Instead of working in the spin basis, it is useful to introduce the helical basis that diagonalizes the single-electron dispersion $\varepsilon_{\sigma \sigma^{\prime}}(\mathbf{k}) \rightarrow\left[U_{\mathbf{k}}^{\dagger} \varepsilon(\mathbf{k}) U_{\mathbf{k}}\right]_{h h^{\prime}}=\left(\epsilon_{\mathbf{k}}+h\left|\boldsymbol{\Gamma}_{\mathbf{k}}\right|\right) \delta_{h h^{\prime}}$ with $h, h^{\prime}= \pm 1$.
1 A. Bentien, S. Johnsen, G. K. H. Madsen, B. B. Iversen, and F. Steglich, European Physics Letters 80, 17008 (2007).

2 P. Ghaemi, R. Mong, and J. E. Moore, Physical Review Letters 105, 166603 (2010).

3 B. Poudel et al., Science 320, 634 (2008).

${ }^{4}$ C. Wood, Rep. Prog. Phys. 51, 459 (1988).

${ }^{5}$ H. Kleinke, Chem. Mater. 22, 604 (2009).

6 P. W. Anderson, Physical Review 124, 41 (1961).

7 A. Hewson, The Kondo problem to heavy fermions (Cambridge, 1993).

8 N. Read, D. Newns, and S. Doniach, Physical Review B 630, 3841 (1984).

9 A. J. Millis and P. A. Lee, Physical Review B 35, 3394 (1987).

10 D. Newns and N. Read, Advances in Physics 36, 799 (1987).

11 J. M. Tomczak, K. Haule, T. Miyake, A. Georges, and G. Kotliar, Phys. Rev. B 82, 085104 (2010).

12 P. Coleman, "Heavy fermions: electrons at the edge of magnetism," in Handbook of Magnetism and Advanced Magnetic Materials., edited by H. Kronmuller and S. Parki (John Wiley and Sons, 2007).
13 G. Aeppli and Z. Fisk, Comm. Condens. Matter 16, 155 (1992).

14 P. Riseborough, Adv. Phys.r 49, 257 (2000).

15 K. V. Samokhin, Ann. Phys. 324, 2358 (2009).

16 L. Isaev, D. F. Agterberg, and I. Vekhter, Physical Review B 85, 081107(R) (2012).

17 P. Ghaemi, T. Senthil, and P. Coleman, Physical Review B 77, 245108 (2008).

18 W. Mao and K. Bedell, Physical Review B 59, R15590 (1999).

19 N. M. Ashcroft and N. D. Mermin, Solid State Physics (1976).

20 H. Kontani, Journal of Physical Society of Japan 73, 515 (2004).

21 C. Sanchez-Castro, Philos. Mag. B 73, 525 (1996).

22 A. Georges and G. Kotliar, Physical Review B 45, 6479 (1992).

23 B. S. Shatry, Rep. Prog. Phys. 72, 016501 (2009).

24 M. Dzero, K. Sun, V. Galitski, and P. Coleman, Phys. Rev. Lett. 104, 106408 (2010).

25 G. Beni, Phys. Rev. B 10, 2186 (1974).

26 S. Mukerjee, Phys. Rev. B 72, 195109 (2005).

27 M. Lee et al., Nature Materials 5, 537 (2006).

28 S. Mukerjee and J. Moore, Applied Physics Letters 90, 112107 (2007). 